\title{
Use of Bao Gui capsule in treatment of a polycystic ovary syndrome rat model
}

\author{
YULING LIAN $^{1,2}$, FANGUI ZHAO ${ }^{1,2}$ and WENJUN WANG ${ }^{1,2}$ \\ ${ }^{1}$ Obstetrics and Gynecology Hospital, Fudan University; ${ }^{2}$ Shanghai Key Laboratory of Female Reproductive \\ Endocrine Related Diseases, Obstetrics and Gynecology Hospital, Fudan University, Shanghai 200011, P.R. China
}

Received January 31, 2018; Accepted November 16, 2018

DOI: $10.3892 / \mathrm{mmr} .2020 .10953$

\begin{abstract}
The present study was designed to elucidate the underlying mechanisms of Bao Gui capsule (BGC) against hyperandrogenism, insulin resistance and leptin resistance of PCOS. Letrozole was used to induce a PCOS model in rats, which were then randomly divided into four groups $(n=9)$ : Control, Model, high-dose BGC (BGC-H) and low-dose BGC (BGC-L) group. Serum levels of follicle-stimulating hormone (FSH), luteinizing hormone ( $\mathrm{LH})$, testosterone (T), estradiol $\left(\mathrm{E}_{2}\right)$, insulin, leptin, and interleukin (IL)-1 $\beta$, IL-6 and tumor necrosis factor- $\alpha$ (TNF- $\alpha)$ in the hypothalamus were determined by ELISA. Protein levels of cytochrome P450c17 $\alpha$ and cytochrome P450 aromatase (P450arom) in ovaries were determined by immunohistochemistry and western blot analysis. Additionally, the expression of GLUT4 in uterus and muscle tissue, and NF- $\mathrm{BB}, \mathrm{IKK} \beta$ and SOCS $3 \mathrm{mRNA}$ levels in the hypothalamus were evaluated. BGC significantly reduced body weight gain and decreased serum levels of LH/FSH, T, $\log \mathrm{T} / \mathrm{E}_{2}$, insulin and leptin compared with the PCOS model rats. Furthermore, BGC markedly reduced the expression of $\mathrm{P} 450 \mathrm{c} 17 \alpha$ and significantly increased the expression of P450arom in ovaries, and increased the expression of GLUT4 in uterus and muscle tissues. BGC also effectively reduced the level of IL- 6 and TNF- $\alpha$, and the expression of IKK $\beta, N F-\kappa B$ and SOCS3 in the hypothalamus of PCOS model rats. These results suggest that BGC may effectively improve hyperandrogenism, insulin resistance, endometrial receptivity and the low-grade chronic inflammation in the hypothalamus.
\end{abstract}

Correspondence to: Dr Wenjun Wang, Obstetrics and Gynecology Hospital, Fudan University, 128 Shenyang Road, Shanghai 200011, P.R. China

E-mail: fduwjwwang@163.com

Abbreviations: PCOS, polycystic ovary syndrome; GLUTs, glucose transporters; P450arom, cytochrome P450 aromatase; BGC, Bao Gui capsule

Key words: polycystic ovary syndrome, traditional Chinese medicine, Bao Gui capsule

\section{Introduction}

Polycystic ovary syndrome (PCOS) is the most common endocrinological disorder and affects $12-21 \%$ of reproductive-aged women with reproductive, cardiometabolic, and psychological impacts; however, the mechanism of PCOS development remains unclear (1-3). It is well established that the major cause of PCOS pathogenesis, and its associated metabolic disorders, is hyperandrogenism. A previous study demonstrated that abnormal expression of cytochrome $\mathrm{P} 450 \mathrm{c} 17 \alpha$ and cytochrome $\mathrm{P} 450$ aromatase (P450arom) has a vital role in local ovarian hyperandrogenism during PCOS (4). Since insulin resistance is a common feature of women with PCOS, it is in turn associated with compensatory hyperinsulinemia, which has an important role in determining androgen excess production rates. A large number of researches indicate that glucose transporters (GLUTs) are responsible for transport of glucose across the plasma cell membrane, which is the rate-limiting step in glucose metabolism $(5,6)$. Recent studies have demonstrated that the changes in GLUT4 expression or translocation in the uterus or muscle tissues are involved in insulin resistance during PCOS $(7,8)$. In addition, leptin, which was the first adipokine to be discovered, has a crucial role in maintaining food intake and energy balance, and regulates reproduction function (9-11). Our previous study suggested that there was a state of chronic low-grade inflammation in the hypothalamus of PCOS model rats, which may account for central leptin resistance rats (12). The exact mechanism of PCOS remains unclear and requires further research.

Treatment for PCOS involves hormone therapy, ovulation induction and surgery. Western treatment of PCOS can promote ovulation, normalize endocrine metabolism, and improve clinical symptoms $(13,14)$. However, drug dependence or relapse following use of drugs and toxic side effects of oral medication greatly limit the clinical use of western medicine (15). Traditional Chinese medicine (TCM), which originated in China $>3,000$ years ago, is an important feature of complementary medicine. From the perspective of TCM, reproductive function is governed by the kidneys. According to TCM, the etiology and pathogenesis of PCOS are associated with disorders of the kidneys, liver and spleen, and kidney deficit (in TCM terms), is viewed as the primary problem in PCOS (16,17). Bao Gui capsule (BGC), a Chinese medicinal formula used to treat PCOS, has been demonstrated to 
improve hyperandrogenism and insulin resistance in clinical practice $(18,19)$. According to the TCM theory, BGC has an important role in the special therapeutic method of "nourishing kidney, strengthening spleen, removing dampness, dissolving phlegm and eliminating blood stasis' $(18,19)$. Sun and Yu $(20)$ reported that $\mathrm{BGC}$ has an important role in central regulation of proopiomelanocortin, neuropeptide $\mathrm{Y}$ and leptin receptor, and reduces serum leptin levels in androgen-sterilized rat at 9 days old. Although the mechanism of BGC in treating PCOS remains unclear, studies on patients and animal model in the past years have demonstrated that BGC may have a role in regulating the neuro-endocrine-metabolic network $(19,21)$. Kuek et al (22) report that the effects of BGC on hyperandrogenism are not as marked as Diane-35, but more effective than metformin. The effects of BGC on hyperinsulinemia are not as marked as metformin, but more effective than Diane-35.

The current study was undertaken to observe the effect of BGC on the expression of P450arom and P450c17 $\alpha$ in ovarian tissues, and the expression of GLUT4 in uterus and muscle tissue of rats in a PCOS model. Furthermore, regarding the effect of low-grade chronic inflammation on leptin resistance in PCOS rats, expression of interleukin (IL)-1 $\beta$, IL-6, tumor necrosis factor- $\alpha$ (TNF- $\alpha$ ) and nuclear factor- $\kappa \mathrm{B}$ kinase subunit $\beta(\mathrm{IKK} \beta) /$ nuclear factor- $\kappa \mathrm{B}(\mathrm{NF}-\kappa \mathrm{B})$ in the hypothalamus was determined.

\section{Materials and methods}

Drugs and preparation. BGC was obtained from the Obstetrics and Gynecology Hospital of Fudan University (Shanghai, China), and is patented and approved by Shanghai Food and Drug Administration (no. YZ120296). BGC is composed of Herba Epimedium, Rhizoma Polygonati, Fructus Psoraleae, Carapax et Plastrum Testudinis, Radix Rehmanniae, Rhizoma Anemarrhenae, Radix Angelicae Sinensis, Semen Persicae, Rhizoma Acori Tatarinowii, Radix Polygoni Cuspidati, Herba Verbenae Officinalis and Radix Ophiopogonis. It is a hospital prescription which was produced by Cai Tong De Shanghai Pharmaceutical Co. Ltd. (http://www.ctdtzy.com/) and termed Tian Gui Capsule. In 2012 it was renamed Bao Gui Capsule and produced by Fang Xin Shanghai Pharmaceutical Co. Ltd. (www.fangxinhealth.com). The ingredients of the BGC capsule were carefully analyzed and quality-controlled by the manufacturer. Each capsule weighed $0.3 \mathrm{~g}$, which is equivalent to $3.75 \mathrm{~g}$ of crude drug. According to the clinical dosage of $5.4 \mathrm{~g} / 60 \mathrm{~kg} / \mathrm{day}$, the corresponding dose of BGC tablet for rats was $0.567 \mathrm{~g} / \mathrm{kg}$ per day (23). The BGC powder was suspended in solvent [1\% carboxymethyl cellulose sodium (CMC-Na)] and stored at $4^{\circ} \mathrm{C}$ prior to subsequent use. In the current study, rats in the low dose (BGC-L) and high dose (BGC-H) groups received 0.28 and $0.57 \mathrm{~g} / \mathrm{kg} / \mathrm{day} \mathrm{BGC}$ by oral gavage once daily for 3 consecutive weeks.

Animals. Inbred female Sprague-Dawley rats $(n=39$; 6-weeks-old; specific-pathogen free; body weight, 220-240 g) were purchased from Shanghai Jie Esprit Laboratory Animal Technology Co., Ltd. [Shanghai, China; animal license no. SCXK (Shanghai) 2013-0006, http://www.jsj-lab.com]. Rats were housed and experiments were performed at Shanghai Gynecology and Obstetrics
Hospital of Fudan University (Shanghai, China). Rats were housed in a temperature-controlled room with a $12 / 12$ h light-dark cycle, with ad libitum access to food and water in their cages. All experiments in the current study followed the Criteria of the Medical Laboratory Animal Administrative Committee of Shanghai and the Guide for Care and Use of Laboratory Animals (http://www.shanghai. gov.cn/nw2/nw2314/nw2319/nw2407/nw26170/u26aw27198. html), and were approved by the Institutional Experimental Animals Review Board of Shanghai Gynecology and Obstetrics Hospital, Fudan University (No. 20130215).

Grouping and treatment. Fig. 1 presents a schematic diagram illustrating the design of the experiment. After 3 days of acclimatization, 30 rats were administered a gavage of $1.0 \mathrm{mg} / \mathrm{kg}$ of letrozole (HengRui Pharmaceutical Factory, Jiangsu, China, https://www.hrs.com.cn/index.html) solution once daily for 21 consecutive days to establish the rat model of PCOS, while the other 9 rats (as the Control group) were treated with an equal volume of CMC-Na daily for 21 days. Vaginal smears of rats were taken to determine the successful generation of the PCOS model rats. The disordered estrous cycle of rats indicated a successful PCOS rat model. PCOS was successfully induced in 27 rats, which were randomly divided into three groups as follows: Model group $(n=9)$, BGC-L $(n=9)$ and BGC-H $(n=9)$. Rats in the BGC-L and BGC-H group received 0.28 and $0.57 \mathrm{~g} / \mathrm{kg} /$ day of BGC by oral gavage for 21 days. Rats in the Model and Control group were given an equal volume of CMC-Na for 21 days. Body weight and vaginal smears were recorded daily.

Specimen collection. At the end of the 3-week treatment, all rats were anaesthetized with intraperitoneal injection of pentobarbital $(40 \mathrm{mg} / \mathrm{kg})$ and blood $(\sim 5-7 \mathrm{ml})$ was rapidly drawn from the hearts following an overnight fast. The serum samples were stored at $-20^{\circ} \mathrm{C}$. Following euthanasia with an overdose of pentobarbital sodium $(200 \mathrm{mg} / \mathrm{kg})$, the brain were rapidly dissected on ice, then flash frozen in liquid nitrogen. The hypothalamic region extending from the organum vasculosum of the lamina terminalis to the arcuate nucleus was microdissected, and then stored at $-80^{\circ} \mathrm{C}$. Ovary, uterus and gastrocnemius tissues of each group were dissected, with half of each sample immediately put into $4 \%$ paraformaldehyde solution for immunohistochemical analysis, and the remaining tissue stored at $-80^{\circ} \mathrm{C}$ for subsequent western blot analysis.

ELISA. Four hypothalamus tissues of each group were collected, and total proteins were extracted in radioimmunoprecipitation assay (RIPA) buffer (Cell Signaling Technology, Inc., Danvers, MA, USA) following the manufacturer's instructions. Serum levels of follicle-stimulating hormone (FSH), luteinizing hormone $(\mathrm{LH})$, testosterone $(\mathrm{T})$, estradiol $\left(\mathrm{E}_{2}\right)$, fasting insulin (INS) and leptin, and IL- $1 \beta$, IL-6, TNF- $\alpha$ concentration in hypothalamus tissues, were detected using ELISA kits according to the manufacturer's protocols (Table S1).

Immunohistochemistry. The samples were fixed in $4 \%$ paraformaldehyde over $24 \mathrm{~h}$ at $4^{\circ} \mathrm{C}$ prior to embedding in paraffin blocks, and sliced into $4 \mu \mathrm{m}$ thick sections. Following routine deparaffinization in xylene, and rehydration in a graded 


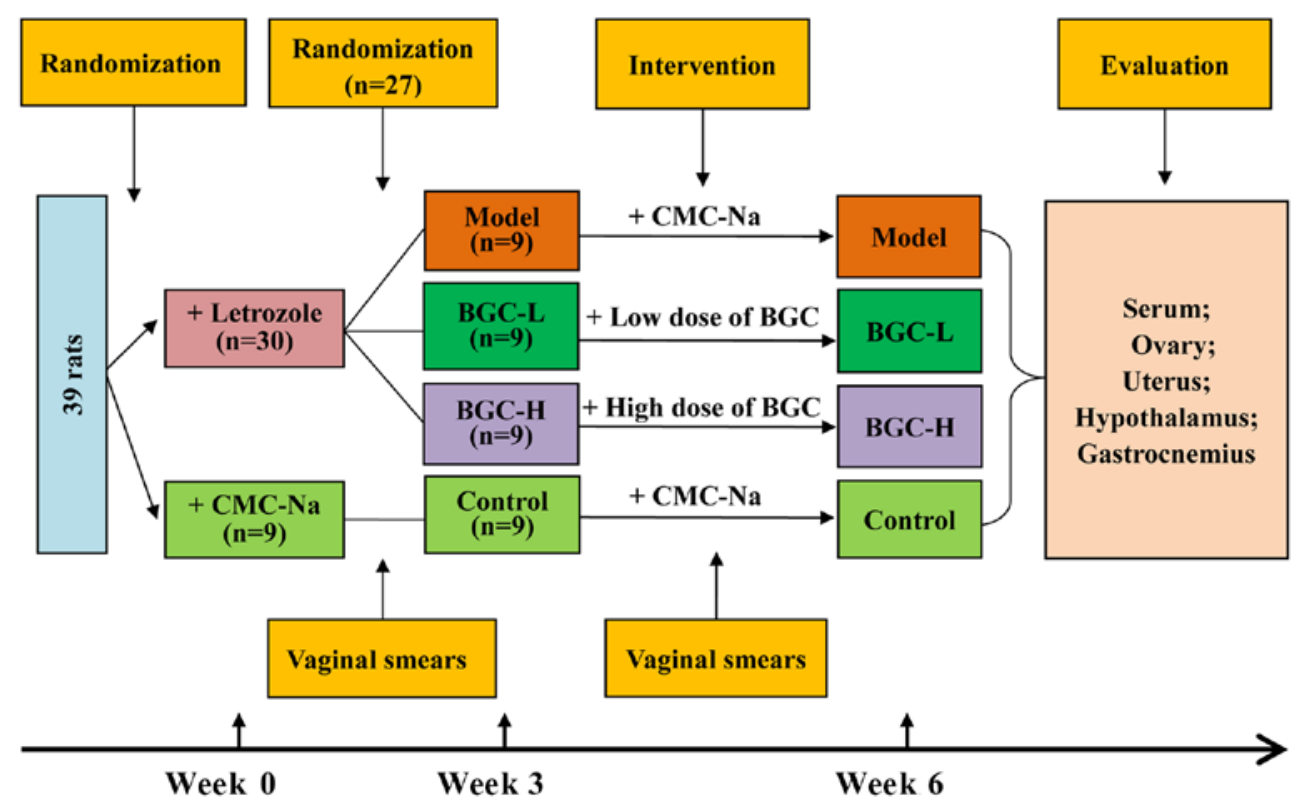

Figure 1. Flow chart of the experiment. CMC-Na, carboxymethyl cellulose sodium; BGC, Bao Gui capsule; BGC-L, low dose; BGC-H, high dose.

ethanol series, the sections were immersed in hematoxylin staining solution (C0107, Beyotime Institute of Biotechnology) for $5 \mathrm{~min}$ at room temperature. Following rinsing with distilled water, sections were immersed in Eosin staining solution (C0109, Beyotime Institute of Biotechnology) for $1 \mathrm{~min}$ at room temperature. Then the sections were dehydrated in ethanol, and mounted with coverslips for pathological confirmation under a light microscope. Tissue slides were subjected to antigen retrieval using citric acid $(0.1 \mathrm{~mol} / \mathrm{l})$ by being immersed in citric acid and boiling for 15 min using an $800 \mathrm{~W}$ microwave oven, and then were left to cool naturally at room temperature before being washed well in phosphate-buffered saline (PBS) $(10 \mathrm{mmol} / 1, \mathrm{pH}$ 7.4). The rabbit polyclonal antibodies against P450c17 $\alpha$ (cat. no. ab125022; Abcam, Cambridge, MA, USA; 1:100), P450arom (cat. no. ab18995; Abcam; 1:200), and GLUT4 (cat. no. ab654; Abcam; 1:100) used as primary antibodies. Sections were incubated with primary antibody overnight at $4^{\circ} \mathrm{C}$, and then biotinylated secondary antibody (cat. no. MR-M100; MingRui BioTech, Co., Ltd., Shanghai, China; 1:300) for $30 \mathrm{~min}$ at room temperature. Diaminobenzidine staining was performed until appropriate for microscopic examination. The primary antibody was replaced with PBS for negative control slides. Immunoreactivity staining was characterized quantitatively by digital image analysis using the Image Pro-Plus 6.0 (Media Cybernetics, Inc., Rockville, MD, USA).

Western blot analysis. Following the manufacturer's instructions, total proteins were extracted using RIPA (Cell Signaling Technology, Inc.) and protein concentrations were determined using bicinchoninic acid assay (Beyotime Institute of Biotechnology, Haimen, China). Following denaturation, protein $(40 \mu \mathrm{g})$ was separated by SDS-PAGE on $12 \%$ gels and then transferred onto a nitrocellulose membrane (EMD Millipore, Billerica, MA, USA), which were blocked in 5\% skimmed milk for $1 \mathrm{~h}$ at room temperature. The membranes were incubated overnight at $4^{\circ} \mathrm{C}$ with specific primary antibodies. The primary antibody dilutions were 1:1,000 for antibodies against P450c17 $\alpha$ and P450arom, and 1:5,000 for GADPH (cat. no. ab181603; Abcam). The membranes were subsequently incubated with a horseradish peroxidase (HRP)-conjugated secondary antibody $(1: 10,000,4414$, Cell Signaling Technology, Inc.) for $1 \mathrm{~h}$ at room temperature. Antibody binding was detected by enhanced chemiluminescence using a Prolight HRP western blotting detection reagent (cat. no. WBKLS0100; Merck KGaA, Darmstadt, Germany). Relative band intensities were analyzed using Image Lab software (version 4.0; Bio-Rad Laboratories, Inc., Hercules, CA, USA).

Reverse transcription-quantitative polymerase chain reaction (RT-qPCR). Total RNA was extracted from hypothalamus tissue using TRIzol reagent (Invitrogen; Thermo Fisher Scientific, Inc., Waltham, MA, USA) according to the manufacturer's instructions. Purified RNA samples were resuspended in diethypyrocarbonate water. Then RNA was reverse transcribed in $20 \mu 1$ total volume using the Reverse Transcription Reagent kit (Takara Bio, Inc., Dalian, China) for $60 \mathrm{~min}$ at $42^{\circ} \mathrm{C}$ and $5 \mathrm{~min}$ at $70^{\circ} \mathrm{C}$. qPCR was performed in an ABI PRISM 7000HT system (Applied Biosystem; Thermo Fisher Scientific, Inc.) in triplicates using the SYBR Green Master Mix (Takara Bio, Inc.). The primers sequences used are presented in Table I. The PCR conditions were as follows: $15 \mathrm{sec}$ at $95^{\circ} \mathrm{C}, 1 \mathrm{cycle} ; 5 \mathrm{sec}$ at $95^{\circ} \mathrm{C}$ and $34 \mathrm{sec}$ at $60^{\circ} \mathrm{C}$, 40 cycles. $\beta$-actin served as the internal control. The $2^{-\Delta \Delta C q}$ method was used to calculate the relative mRNA levels (24).

Statistical analysis. Data are expressed as the mean \pm standard deviation. The comparison among four groups was analyzed by one-way analysis of variance. The least significant difference test was performed to compare the differences between two groups. All computations were performed using SPSS 17.0 (SPSS, Inc., Chicago, IL, USA). P $<0.05$ was considered to indicate a statistically significant difference. 
Table I. Primer sequences of target genes.

\begin{tabular}{llc}
\hline Target gene & \multicolumn{1}{c}{ Primer sequences } & Length (bp) \\
\hline NF- $\kappa$ B & 5'-TCTTCGACTACGCGGTTACGG-3' & 21 \\
& 5'-CTCACGAGCTGAGCATGAAGG-3' & 21 \\
SOCS3 & 5'-TCAACGGTCACCTGGACTCCTA-3' & 22 \\
IKK $\beta$ & 5'-GGTCCAGGAACTCCCGAATG-3' & 20 \\
$\beta$-actin & 5'-GCACCCTGGCCTTTGAATG-3' & 19 \\
& 5'-TCCGTTCAAGTCCTCGCTAACA-3' & 22 \\
\hline
\end{tabular}

$\mathrm{NF}-\kappa \mathrm{B}$, nuclear factor- $\mathrm{B}$; SOCS3, suppressor of cytokine signaling 3; IKK $\beta$, nuclear factor- $\kappa \mathrm{B}$ kinase subunit $\beta$.

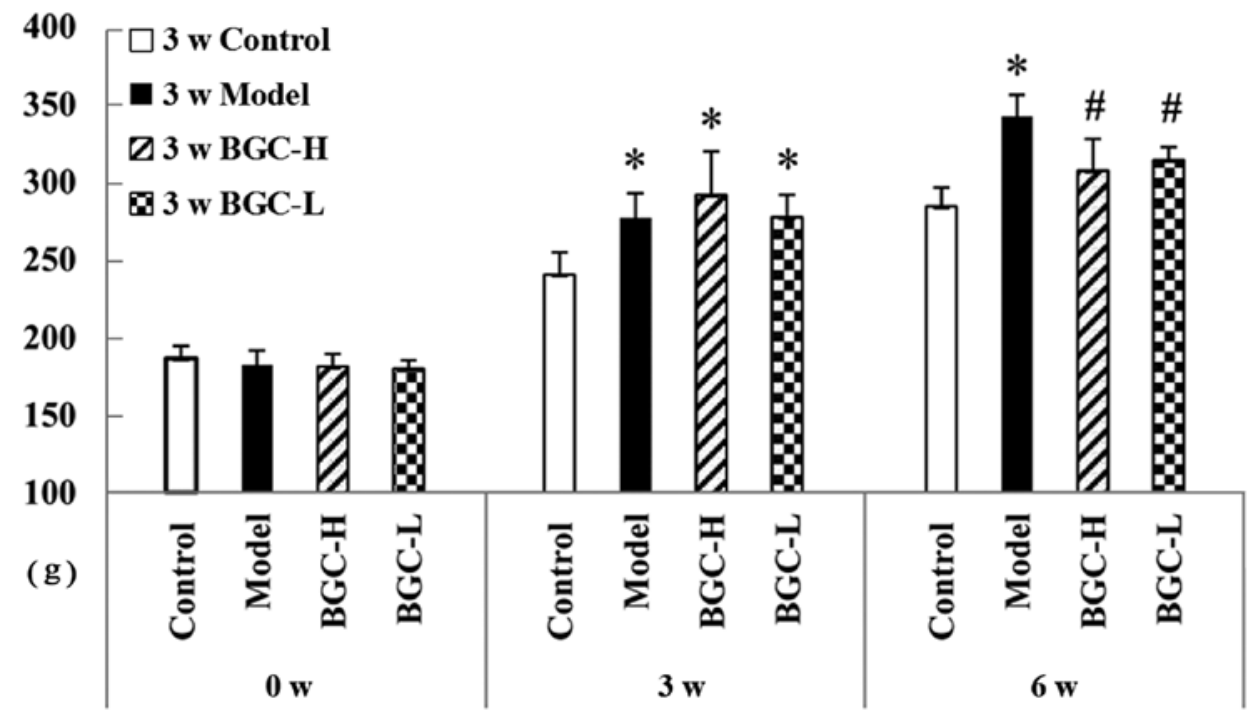

Figure 2. Effect of BGC on the changes of rat body weight. There was no difference in body weight among the four groups prior to treatment (P>0.05). Rats treated with letrozole (Model, BGC-H, BGC-L) gained significantly more body weight than those treated with carboxymethyl cellulose sodium (control) after 3 weeks $(\mathrm{P}<0.05)$. Treatment with either dose of $\mathrm{BGC}$ significantly decreased body weight compared to those in Model group on the sixth week $(\mathrm{P}<0.05)$. Data are expressed as mean \pm standard deviation $(\mathrm{n}=9),{ }^{*} \mathrm{P}<0.05$ vs. Control group. ${ }^{\#} \mathrm{P}<0.05$ vs. Model group. BGC, Bao Gui capsule; BGC-L, low dose; BGC-H, high dose.

\section{Results}

Effect of BGC treatment on rat body weight and histology of ovaries. As demonstrated in Fig. 2, there was no difference in body weight among the four groups before treatment $(\mathrm{P}>0.05)$. Rats treated with letrozole gained significantly more body weight than those treated with $\mathrm{CMC}-\mathrm{Na}$ after 3 weeks $(\mathrm{P}<0.05)$. Treatment with either dose of BGC significantly decreased body weight compared with those in the Model group at week $6(\mathrm{P}<0.05)$.

Under light microscopy, follicles of different developmental stages and a few corpora lutea were observed in the Control group (Fig. 3A). In the PCOS group, there were many follicles with saccular dilatation, and fewer layers of granular cells and thickening surface albuginea (Fig. 3B). The ovaries of rats treated with BGC exhibited increased granular cell layers, decreased dilated follicles, and increased corpus luteum compared with the PCOS model group (Fig. 3C and D).
Effect of BGC treatment on the serum levels of LH/FSH, T, $\log T / E_{2}$, insulin and leptin, and the estrous cycle. Serum $\mathrm{LH} / \mathrm{FSH}, \mathrm{T}, \log \mathrm{T} / \mathrm{E}_{2}$, insulin and leptin concentrations were significantly higher in the Model group compared with the Control group $(\mathrm{P}<0.05$; Fig. 4A-E). Compared with the Model group, the serum levels of LH/FSH, T, $\log \mathrm{T} / \mathrm{E}_{2}$, insulin and leptin were significantly reduced in rats treated with the low and high dose of BGC $(\mathrm{P}<0.05$; Fig. 4A-E). The results of vaginal smears indicated that estrous cycles of rat were routine in the Control group, comprising proestrus, estrus, metestrus and diestrus. Rats experienced prolonged diestrus beginning on the day 11 after the letrozole gavage. Of 30 rats, 27 exhibited vaginal keratosis and an irregular estrous cycle at week 3 , suggesting the successful induction of PCOS. Following 6 weeks of letrozole, only 1 of 9 rats in the Model group demonstrated a regular estrous cycle. In the BGC-L and BGC-H groups, 5 and 6 of the 9 rats exhibited recovered estrous cycles, respectively. The recovery rates in the Model, BGC-L, and BGC-H groups were $11.11,55.56$ and $66.67 \%$, respectively (Fig. 4F). 

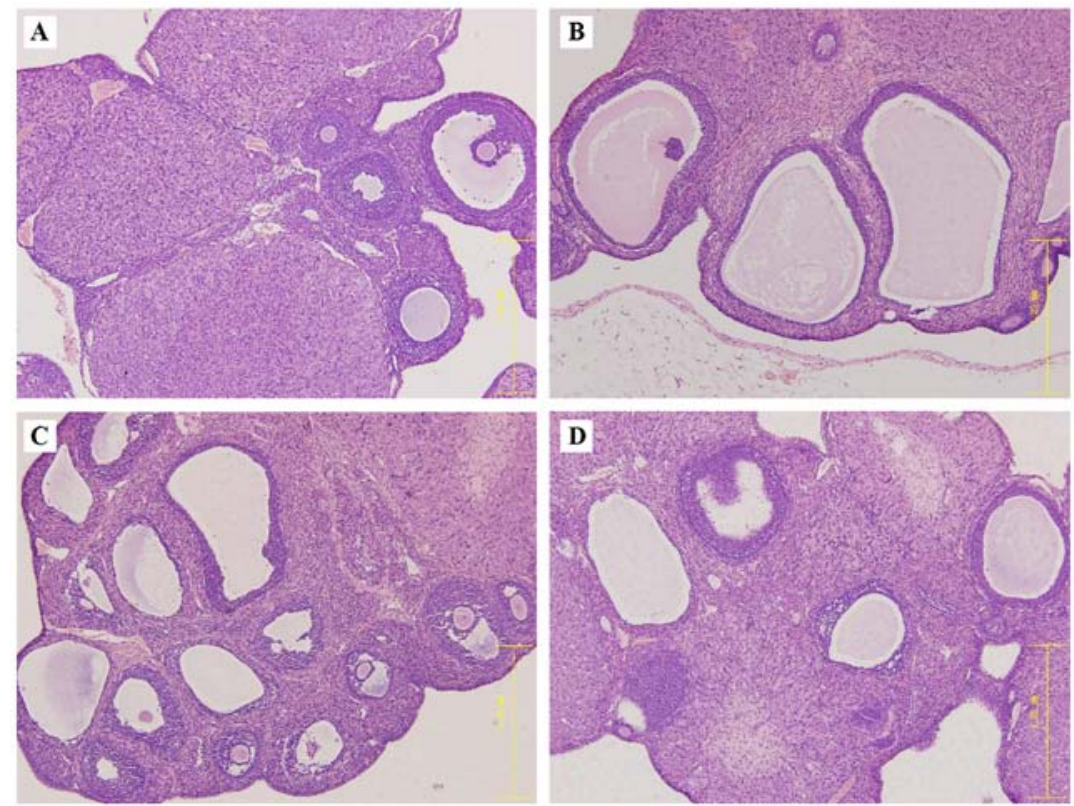

Figure 3. Effects of BGC on histopathological morphology of ovarian tissues. (A) Control, (B) polycystic ovary syndrome model, (C) BGC-H, (D) BGC-L. Ovary tissues were images at x100 magnification. BGC, Bao Gui capsule; BGC-L, low dose; BGC-H, high dose.
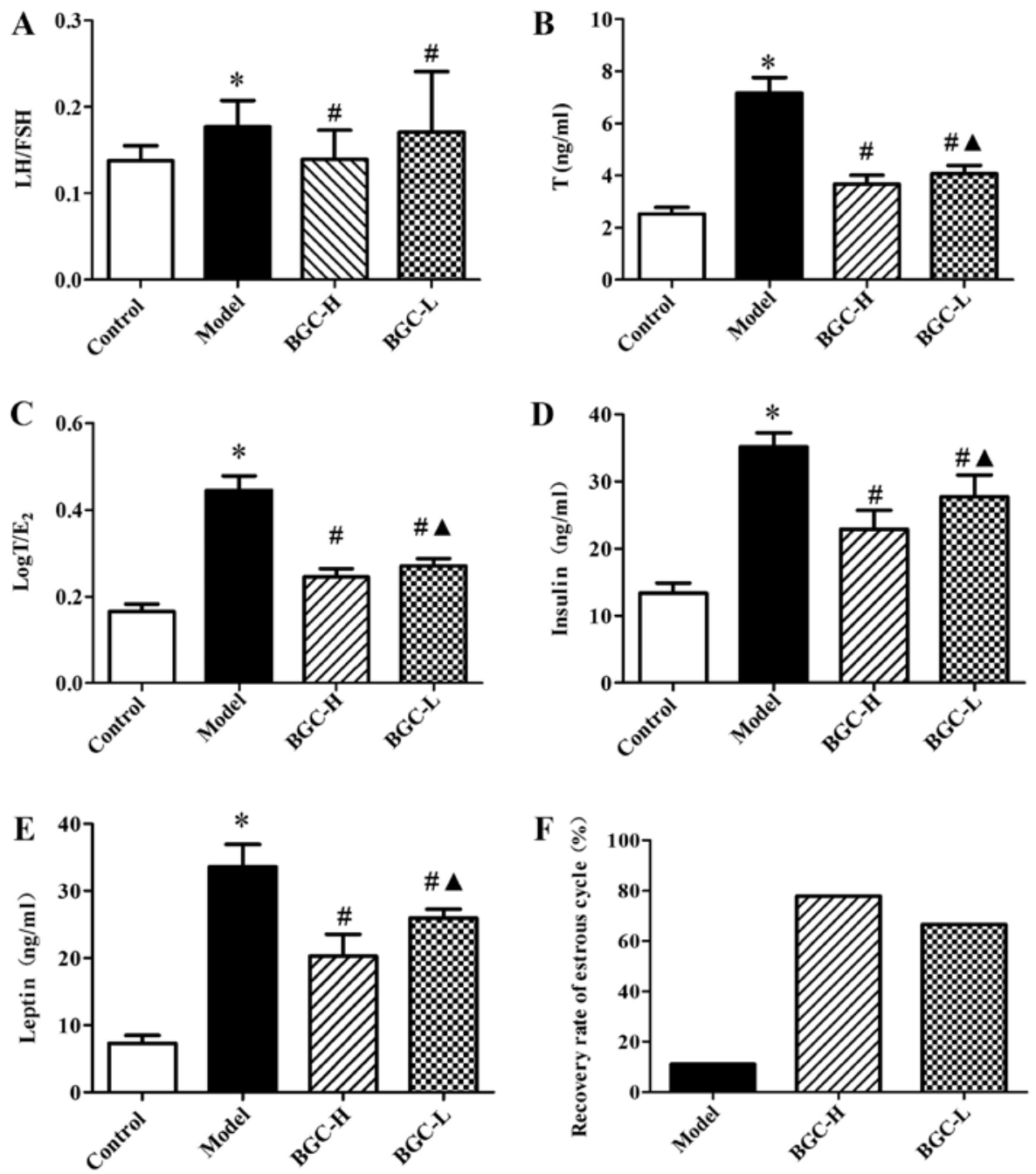

Figure 4. Effects of BGC on serum concentrations of LH/FSH, T, $\log \mathrm{T} / \mathrm{E}_{2}$, insulin, leptin and effects on estrous cycle. Serum levels of (A) LH/FSH, (B) T, (C) $\log \mathrm{T} / \mathrm{E}_{2}$, (D) insulin, (E) leptin and (F) estrous cycle ( $\mathrm{n}=9$ ). Data are expressed as the mean \pm standard deviation $(\mathrm{n}=9)$. ${ }^{*} \mathrm{P}<0.05$ vs. Control group. ${ }^{*} \mathrm{P}<0.05$ vs. Model group. ${ }^{\wedge} \mathrm{P}<0.05$ vs. BGC-H group. FSH, follicle-stimulating hormone; BGC, Bao Gui capsule; BGC-L, low dose; BGC-H, high dose; LH, luteinizing hormone; T, testosterone; $\mathrm{E}_{2}$, estradiol; INS, fasting insulin. 
A
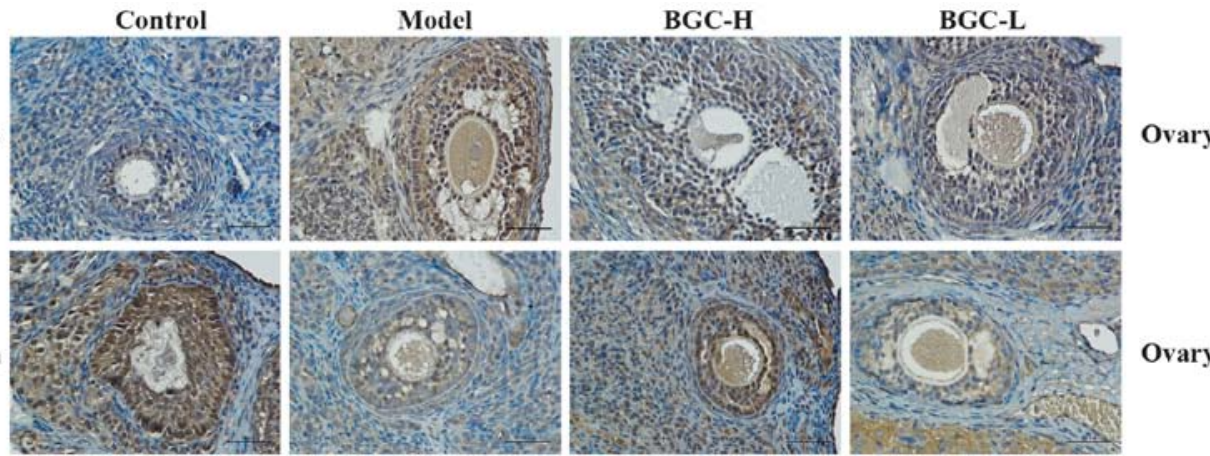

GLUT4
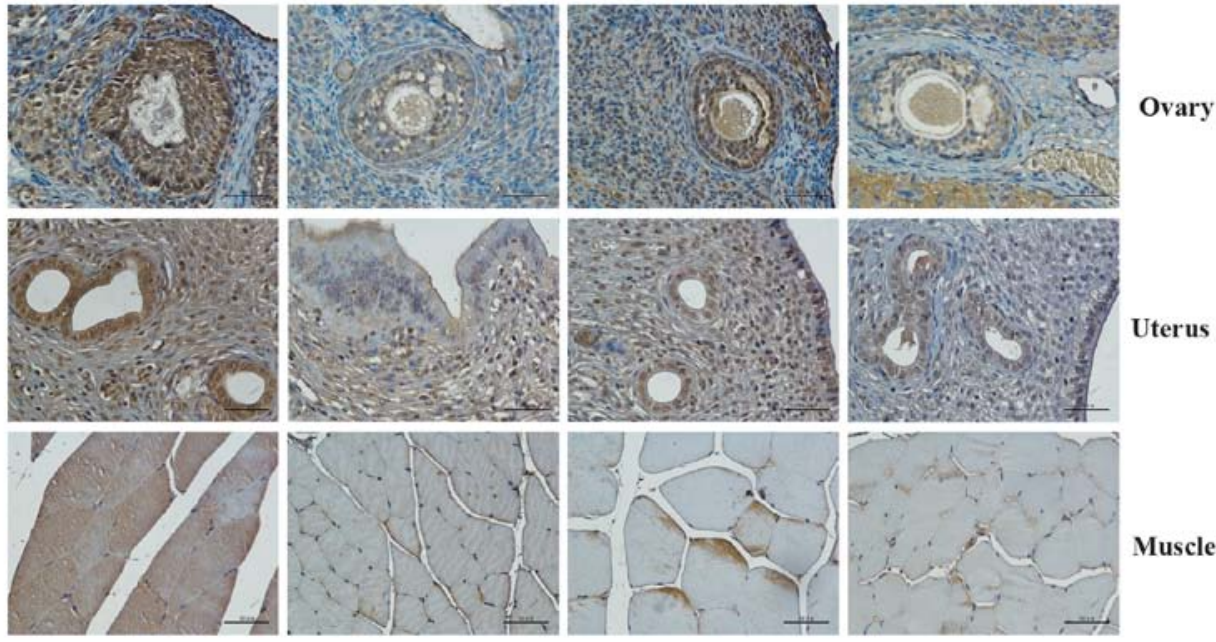

B

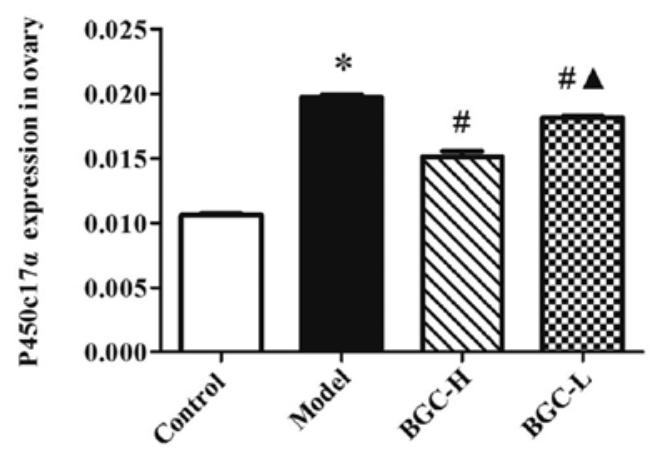

D

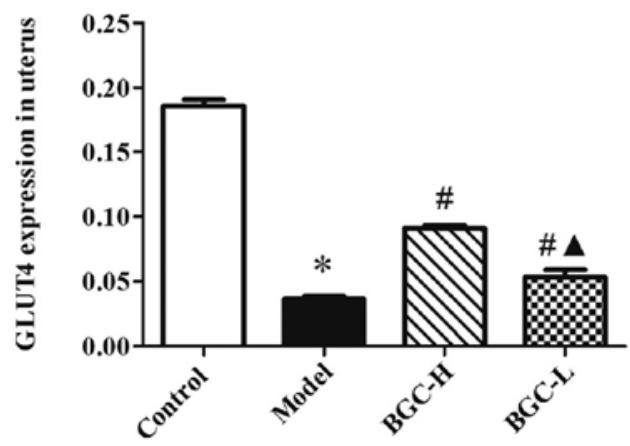

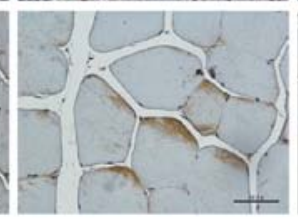

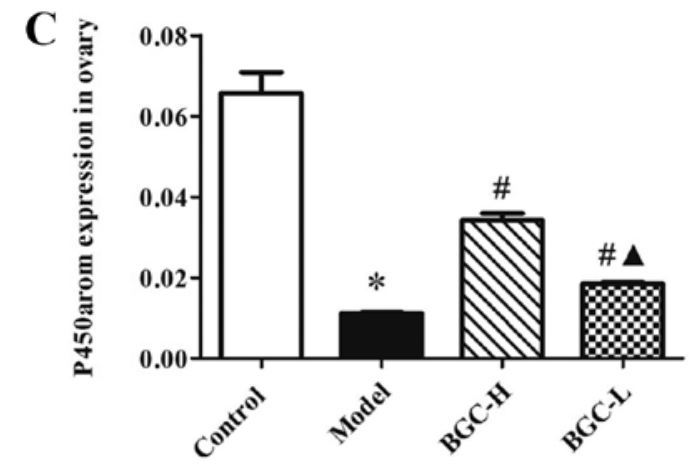

E

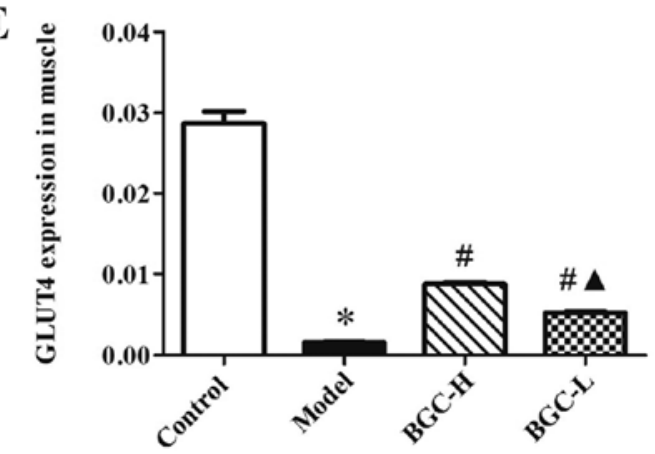

Figure 5. Effects of BGC on the expression of P450c17 $\alpha$ and P450arom in ovarian tissues, and GLUT4 in uterus and muscle tissues analyzed by immunohistochemistry. (A) Immunohistochemical staining of P450c17 $\alpha$ and P450arom in ovarian tissues, and GLUT4 in uterus and muscle tissues (magnification, $\mathrm{x} 400$ ). Statistical analysis of the protein expression of (B) P450c17 $\alpha$ and (C) P450arom in ovarian tissues, and GLUT4 in (D) uterus and (E) muscle tissue.. Data are expressed as the mean \pm standard deviation $(\mathrm{n}=9)$. ${ }^{*} \mathrm{P}<0.05$ vs. Control group. ${ }^{\#} \mathrm{P}<0.05$ vs. Model group. ${ }^{\wedge} \mathrm{P}<0.05$ vs. BGC-H group. BGC, Bao Gui capsule; BGC-L, low dose; BGC-H, high dose; P450c17 $\alpha$, cytochrome P450c17 $\alpha$; P450arom, cytochrome P450 aromatase; GLUT4, glucose transporter 4.

Effect of BGC treatment on the expression of P450c17a and P450arom in ovarian tissues. As presented in Fig. 5A and $\mathrm{B}$, increased immunostaining of $\mathrm{P} 450 \mathrm{c} 17 \alpha$ and decreased immunostaining of P450arom were observed in the ovarian granulosa cells of rats in the Model group compared with rats in the Control group $(\mathrm{P}<0.05)$. Treatment with BGC reduced expression of $\mathrm{P} 450 \mathrm{c} 17 \alpha$ and increased expression of $\mathrm{P} 450$ arom in the ovarian granulosa cells of PCOS model rats $(\mathrm{P}<0.05)$.
Furthermore, the protein levels of P450c17 $\alpha$ and P450arom in the ovarian tissues were assessed via western blot analysis (Fig. 6). The protein expression exhibited a tendency similar to the immunohistochemical staining results.

$B G C$ treatment increased the expression of GLUT4 in uterus and muscle tissues. Compared with the Control group, the expression of GLUT4 in uterus and muscle tissues were 

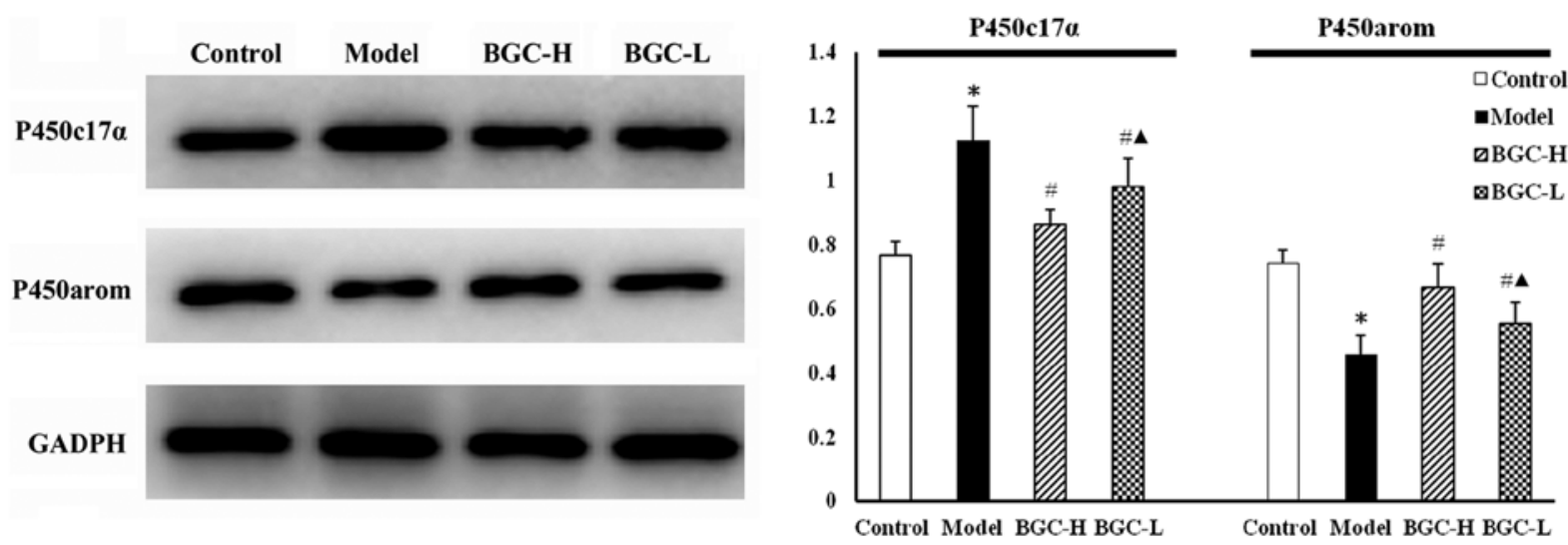

Figure 6. Effects of BGC on the protein expression of $\mathrm{P} 450 \mathrm{c17} \alpha, \mathrm{P} 450$ arom in ovarian tissues. Data are expressed as mean \pm standard deviation $(\mathrm{n}=4),{ }^{*} \mathrm{P}<0.05$ vs. Control group. ${ }^{\#} \mathrm{P}<0.05$ vs. Model group. ${ }^{\wedge} \mathrm{P}<0.05$ vs. BGC-H group. BGC, Bao Gui capsule; BGC-L, low dose; BGC-H, high dose; P450c17a, cytochrome $\mathrm{P} 450 \mathrm{c} 17 \alpha$; P450arom, cytochrome $\mathrm{P} 450$ aromatase.

significantly decreased in the Model group $(\mathrm{P}<0.05)$. The expression of GLUT4 was significantly increased in low dose and high dose BGC treatment groups compared with the Model group ( $\mathrm{P}<0.05$; Fig. 7).

$B G C$ treatment reduces low-grade chronic inflammation in the hypothalamus and may improve central leptin resistance. The protein levels of IL-1 $\beta$, IL- 6 and TNF- $\alpha$ in the hypothalamus were determined via ELISA. The IL-1 $\beta$, IL- 6 and TNF- $\alpha$ concentrations in the hypothalamus were significantly increased in the Model group compared with the Control group $(\mathrm{P}<0.05)$. Compared with the Model group, the protein levels of IL-6 and TNF- $\alpha$ were significantly decreased in BGC-H group in a dose-dependent manner $(\mathrm{P}<0.05$, Fig. 8A-C).

Additionally, NF- $\mathrm{B}, \mathrm{IKK} \beta$ and suppressor of cytokine signaling 3 (SOCS3) mRNA levels in the hypothalamus were examined. NF- $\mathrm{NB}, \mathrm{IKK} \beta$ and SOCS 3 mRNA levels in the hypothalamus were markedly increased in the Model group compared with the Control group, and were significantly decreased in the low dose and high dose BGC treatment groups $(\mathrm{P}<0.05$; Fig. 8D-F).

\section{Discussion}

In the present study, serum $\mathrm{LH} / \mathrm{FSH}, \mathrm{T}, \log \mathrm{T} / \mathrm{E}_{2}$ insulin and leptin concentrations were significantly higher in the Model group compared with the Control group. Despite the observed increase in $\log \mathrm{T} / \mathrm{E}_{2}$ in the Model group, the level of estradiol was observed to be significantly decreased in the Model group compared with the Control group rats (Fig. S1). The species difference of changes of estradiol level between human and rat with PCOS, and the less number of rats in each group are probably the main reason, for the experiment results above. BGC has been used as a prescription since 2006 , and is produced as a patented medicine and widely used as PCOS medication. Clinically, patients usually take BGC for at least three menstrual cycles (23). As the estrous cycle of rats is 4-5 days, BGC administration for three weeks was equivalent to patients taking $\mathrm{BGC}$ for at least three menstrual cycle. In the present study, $\mathrm{BGC}$ reduced $\mathrm{LH} / \mathrm{FSH}, \mathrm{T}, \log \mathrm{T} / \mathrm{E}_{2}$, insulin and leptin. Additionally, BGC markedly reduced body weight gain, decreased the expression of $\mathrm{P} 450 \mathrm{c} 17 \alpha$ in ovaries, significantly increased the expression of P450arom in ovaries, and the expression of GLUT4 in uterus and muscle tissues. Furthermore, BGC effectively reduced the level of IL-1 $\beta$, IL-6 and TNF- $\alpha$, and the expression of IKK $\beta, N F-\kappa B$ and SOCS3 in the hypothalamus of PCOS model rats.

It is established that increased androgen levels, which appear to be the result of dysregulation of steroidogenesis within the ovaries and adrenal glands, is the main pathological feature of PCOS. P450c17 $\alpha$, which possesses 17-hydroxylase and 17, 20-lyase activities, is considered to be the rate-limiting enzyme in the formation of androgens (25-27). P450arom induces the conversion of androstenedione and testosterone into estradiol and estrone in granulosa cells. P450arom-catalysed estrogen synthesis is a key event during the final stage of ovarian follicular development (28). Decreased expression of P450arom or increased expression of $\mathrm{P} 450 \mathrm{c} 17 \alpha$ induces hyperandrogenism in patients with PCOS. In the current study, reduced expression of $\mathrm{P} 450 \mathrm{c} 17 \alpha$ and increased expression of P450arom were observed in ovarian tissues following BCG treatment. Thus, BGC improved the local ovarian environment of excessive androgens by altering the expression of P450arom and $\mathrm{P} 450 \mathrm{c} 17 \alpha$, which may further improve the reproductive, endocrine and metabolic disorder associated with PCOS.

The translocation of GLUT4 vesicles from intracellular deposits to the plasma membrane, which is stimulated by insulin or muscle contractions, has a vital role in glucose homeostasis. In skeletal muscle and adipocytes, dysregulation of GLUT4 translocation is closely associated with insulin resistance. Furthermore, based on the fact that glucose uptake and utilization have a vital role in implantation, embryonic development and pregnancy, an increasing number of studies have that steroid hormone-dependent regulation of GLUT4 expression may be involved in the mechanism of PCOS-induced endometrial disorders of the menstrual cycle and endometrial receptivity $(7,8,29)$. In the present study, BGC treatment significantly increased the expression of GLUT4 in uterus and muscle tissues, which suggests that BGC may improve insulin resistance and endometrial receptivity.

Our previous study revealed central leptin resistance and low-grade chronic inflammation in the hypothalamus 
A

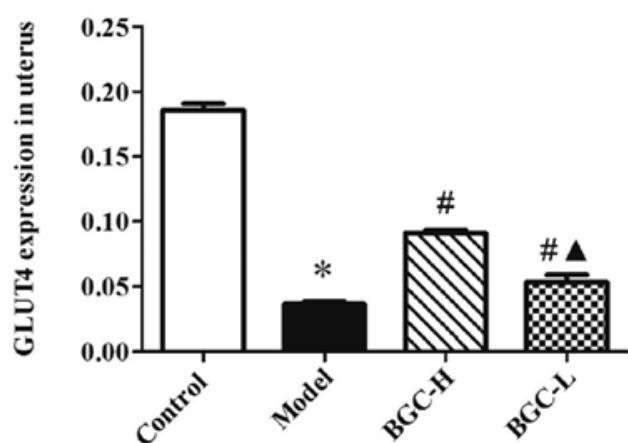

B

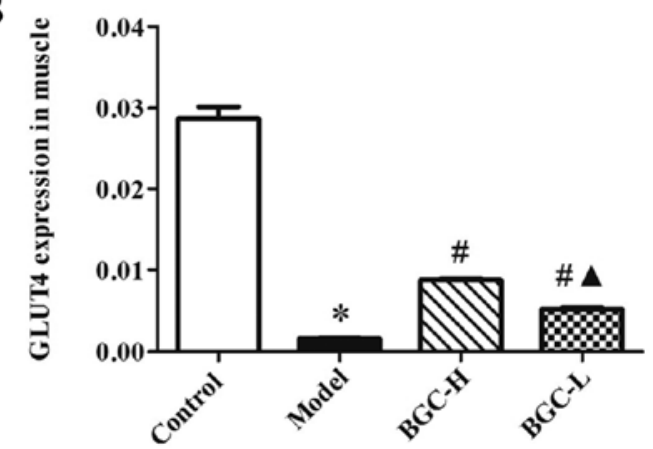

Figure 7. Effects of BGC on the expression of GLUT4. GLUT4 in (A) uterus and (B) muscle tissues. Data are expressed as the mean \pm standard deviation $(\mathrm{n}=9)$. ${ }^{*} \mathrm{P}<0.05$ vs. Control group. ${ }^{~} \mathrm{P}<0.05$ vs. Model group. ${ }^{\wedge} \mathrm{P}<0.05$ vs. BGC-H group. BGC, Bao Gui capsule; BGC-L, low dose; BGC-H, high dose; GLUT4, glucose transporter 4 .
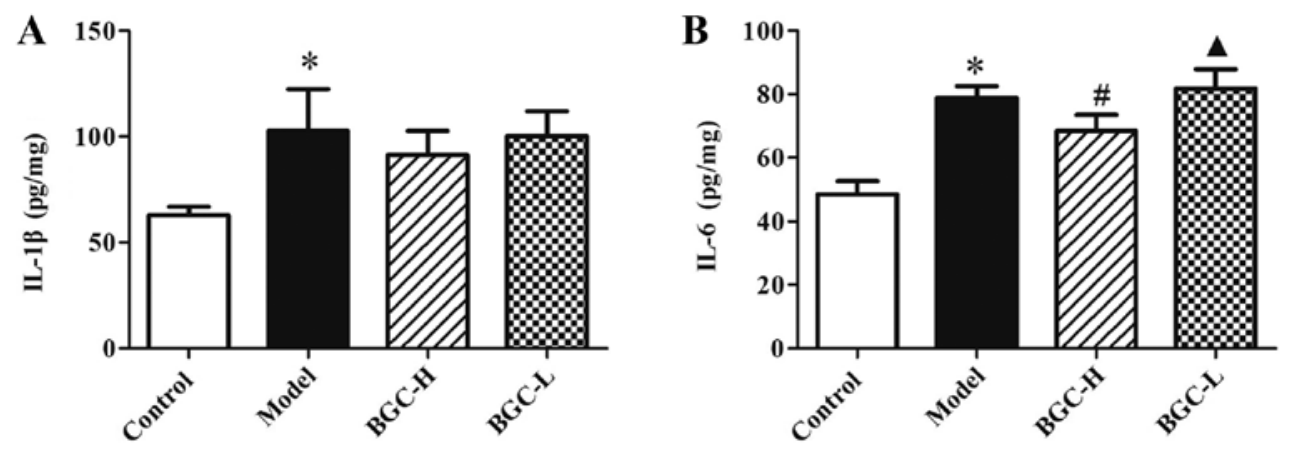

C
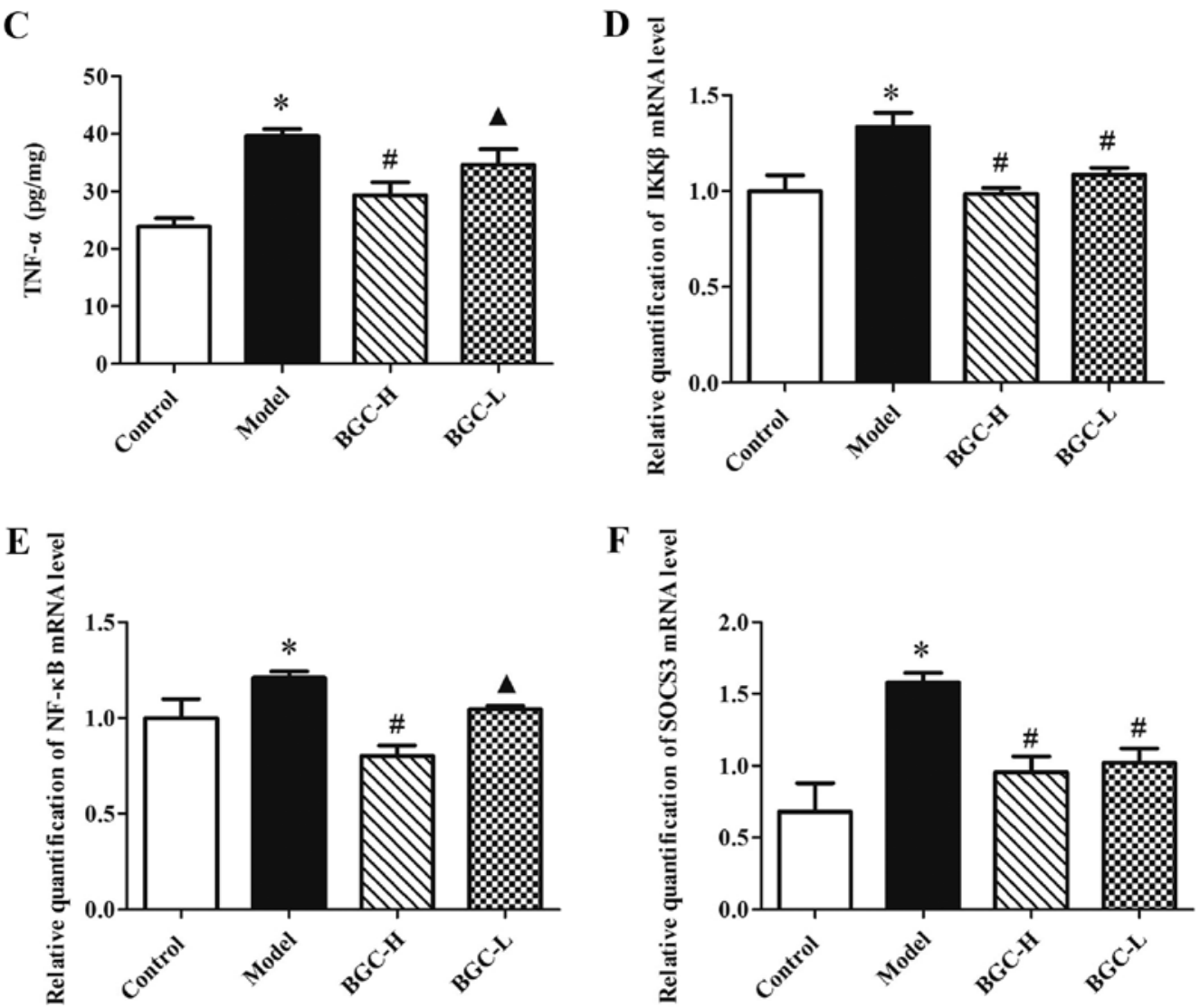

Figure 8. Effects of BGC on protein levels of IL-1 $\beta$, IL- 6 and TNF- $\alpha$ in the hypothalamus, and mRNA expressions of NF- $\mathrm{kB}$, IKK $\beta$ and SOCS3 in hypothalamus. Protein concentrations of (A) IL-1 $\beta$, (B) IL-6 and (C) TNF- $\alpha$ in hypothalamus were determined by ELISA (n=4). (D) IKK $\beta$, (E) NF- $\mathrm{kB}$ and (F) SOCS3 mRNA levels of hypothalamus were determined by reverse transcription-quantitative polymerase chain reaction $(\mathrm{n}=4)$. Data are expressed as the mean \pm standard deviation. ${ }^{*} \mathrm{P}<0.05$ vs. Control group. ${ }^{*} \mathrm{P}<0.05$ vs. Model group. ${ }^{\wedge} \mathrm{P}<0.05$ vs. BGC-H group. IL, interleukin; TNF- $\alpha$, tumor necrosis factor- $\alpha$; IKK $\beta$, nuclear factor- $\kappa \mathrm{B}$ kinase subunit $\beta$; NF- $\mathrm{\kappa B}$, nuclear factor- $\mathrm{\kappa} B$; SOCS3, suppressor of cytokine signaling 3 . 
of PCOS rats (12). In the current study, the protein level of IL-1 $\beta$, IL- 6 and TNF- $\alpha$, and the mRNA expression of IKK $\beta$, $\mathrm{NF}-\kappa \mathrm{B}$ and SOCS3 in the hypothalamus were significantly increased in Model group rats compared with the Control group, which was consistent with our previous conclusions. IKK $\beta / N F-\kappa B$ signaling has been identified as one of the key proinflammatory pathway that atypically mediates chronic low-grade nutritionally-induced inflammation and causes various metabolic dysfunctions (30-32). Recent research indicated that overnutrition activates $I K K \beta / N F-\kappa B$ in the hypothalamus, and furthermore, inhibition of IKK $\beta / N F-\kappa B$ in the hypothalamus suppresses appetite and prevents obesity $(33,34)$. SOCS3 has a crucial role in mediating the effects of hypothalamic IKK $\beta / N F-\kappa B$ on leptin resistance $(35,36)$. Our previous study (12) demonstrated that central icv injection of leptin decreased $24 \mathrm{~h}$ food intake and body weight gain, and induced increasing expression of p-STAT3 in the hypothalamus of control group rats. However, central icv injection of leptin did not exert an effect on $24 \mathrm{~h}$ food intake, body weight, and p-STAT3 expression in rats of PCOS group, suggesting that central leptin resistance was present in the PCOS rats induced by letrozole. Furthermore, inflammatory markers were upregulated in the hypothalami of PCOS rats, indicating that there was a state of chronic low-grade inflammation in the hypothalamus, which may be the potential mechanism of central leptin resistance in PCOS rats (12). In the present study, BGC may have improved central leptin resistance of PCOS rats by decreasing the protein levels of IL- 6 and TNF- $\alpha$, and the mRNA expression of IKK $\beta, N F-\kappa B$ and SOCS3 in hypothalamus of PCOS rats, which may partially explain the therapeutic effects.

In summary, the results of the current study demonstrate that BGC treatment may effectively improve hyperandrogenism by decreasing the expression of $\mathrm{P} 450 \mathrm{c} 17 \alpha$ and raising the expression of $\mathrm{P} 450$ arom in ovarian tissues. Additionally, BGC treatment may improve insulin resistance and endometrial receptivity by increasing the expression of GLUT4 in uterus and muscle tissues. Furthermore, the current study provides the first description that BGC treatment may improve low-grade chronic inflammation in the hypothalamus, and may improve central leptin resistance in PCOS rats. Thus, BGC may be a promising TCM therapy for the treatment of PCOS. Further clinical trials are required to confirm its efficacy in the future.

\section{Acknowledgements}

The authors thank Mr. Pinli Chen from Obstetrics and Gynecology Hospital, Fudan University, Shanghai, China and Dr Xing Tan from Department of Physiology and Center of Polar Medical Research, Second Military Medical University, Shanghai, China for their generous assistance in surgery and the preparation of specimens.

\section{Funding}

The study was supported by a grant from The Science and Technology Commission of Shanghai Municipality (grant no. 11DZ1971900).

\section{Availability of data and materials}

All data generated or analyzed during this study are included in this published article and/or its supplementary materials.

\section{Authors' contributions}

WW conceived and designed the study. YL and FZ performed the experiments. YL wrote the manuscript.

\section{Ethics approval and consent to participate}

All experiments in the current study followed the Criteria of the Medical Laboratory Animal Administrative Committee of Shanghai and the Guide for Care and Use of Laboratory Animals, and were approved by the Institutional Experimental Animals Review Board of Shanghai Gynaecology and Obstetrics Hospital, Fudan University (no. 20130215).

\section{Patient consent for publication}

Not applicable.

\section{Competing interests}

The authors declare that they have no competing interests.

\section{References}

1. March WA, Moore VM, Willson KJ, Phillips DI, Norman RJ and Davies MJ: The prevalence of polycystic ovary syndrome in a community sample assessed under contrasting diagnostic criteria. Hum Reprod 25: 544-551, 2010.

2. Teede HJ, Misso ML, Deeks AA, Moran LJ, Stuckey BG, Wong JL, Norman RJ and Costello MF; Guideline Development Groups: Assessment and management of polycystic ovary syndrome: Summary of an evidence-based guideline. Med J Aust 195: S65-S112, 2011.

3. Moran LJ, Misso ML, Wild RA and Norman RJ: Impaired glucose tolerance, type 2 diabetes and metabolic syndrome in polycystic ovary syndrome: A systematic review and meta-analysis. Hum Reprod Update 16: 347-363, 2010.

4. Qin KN and Rosenfield RL: Role of cytochrome P450c17 in polycystic ovary syndrome. Mol Cell Endocrinol 145: 111-121, 1998.

5. Garvey WT, Maianu L, Hancock JA, Golichowski AM and Baron A: Gene-expression of GLUT4 in skeletal-muscle from insulin-resistant patients with obesity, IGT, GDM, and NIDDM. Diabetes 41: 465-475, 1992.

6. Shepherd PR and Kahn BB: Glucose transporters and insulin action-implications for insulin resistance and diabetes mellitus. N Engl J Med 341: 248-257, 1999.

7. Mioni R, Chiarelli S, Xamin N, Zuliani L, Granzotto M, Mozzanega B, Maffei P, Martini C, Blandamura S, Sicolo N and Vettor R: Evidence for the presence of glucose transporter 4 in the endometrium and its regulation in polycystic ovary syndrome patients. J Clin Endocrinol Metab 89: 4089-4096, 2004.

8. Frolova AI and Moley KH: Glucose transporters in the uterus: An analysis of tissue distribution and proposed physiological roles. Reproduction 142: 211-220, 2011.

9. Trayhurn P and Beattie JH: Physiological role of adipose tissue: White adipose tissue as an endocrine and secretory organ. Proc Nutr Soc 60: 329-339, 2001.

10. Sainz N, Gonzalez-Navarro CJ, Martinez JA and MorenoAliaga MJ: Leptin signaling as a therapeutic target of obesity. Expert Opin Ther Targets 19: 893-909, 2015. 
11. Pusalkar M, Meherji P, Gokral J, Savardekar L, Chinnaraj S and Maitra A: Obesity and polycystic ovary syndrome: Association with androgens, leptin and its genotypes. Gynecol Endocrinol 26: 874-882, 2010

12. Lian Y, Zhao F and Wang W: Central leptin resistance and hypothalamic inflammation are involved in letrozole-induced polycystic ovary syndrome rats. Biochem Biophys Res Commun 476: 306-312, 2016.

13. Prelevic GM, Puzigaca Z and Balint-Peric LA: Effects of an oral contraceptive containing cyproterone acetate (Diane-35) on the symptoms, hormone profile, and ovarian volume of hirsute women with polycystic ovarian syndrome. Ann N Y Acad Sci 687: 255-262, 1993 .

14. Palomba S, Falbo A, Russo T, Orio F, Tolino A and Zullo F: Systemic and local effects of metformin administration in patients with polycystic ovary syndrome (PCOS): Relationship to the ovulatory response. Hum Reprod 25: 1005-1013, 2010.

15. Spritzer PM, Motta AB, Sir-Petermann T and DiamantiKandarakis E: Novel strategies in the management of polycystic ovary syndrome. Minerva Endocrinol 40: 195-212, 2015.

16. Raja-Khan N, Stener-Victorin E, Wu X and Legro RS: The physiological basis of complementary and alternative medicines for polycystic ovary syndrome. Am J Physiol Endocrinol Metab 301: E1-E10, 2011.

17. Zhou J and Qu F: Treating gynaecological disorders with traditional Chinese medicine: A review. Afr J Tradit Complement Altern Med 6: 494-517, 2009.

18. Zhou LR and Yu J: Clinical observation on treatment of hyperinsulinemia and hyperandrogenism anovulatory patient with replenishing kidney-yin drugs. Zhongguo Zhong Xi Yi Jie He Za Zhi 16: 515-518, 1996 (In Chinese).

19. Hou J, Yu J and Wei M: Study on treatment of hyperandrogenism and hyperinsulinism in polycystic ovary syndrome with Chinese herbal formula 'tiangui fang'. Zhongguo Zhong Xi Yi Jie He Za Zhi 20: 589-592, 2000 (In Chinese).

20. Sun F and Yu J: Effect of tiangui recipe on serum leptin and pituitary gonadotropin in androgen-sterilized rats. Zhongguo Zhong Xi Yi Jie He Za Zhi 19: 350-352, 1999 (In Chinese).

21. Yu J and Cheng HY: Experimental studies on the relation of the kidney and reproduction. Zhong Xi Yi Jie He Za Zhi 9: 548-551, 517, 1989 (In Chinese).

22. Kuek S, Wang WJ and Gui SQ: Efficacy of Chinese patent medicine Tian Gui Capsule in patients with polycystic ovary syndrome: A randomized controlled trial. Zhong Xi Yi Jie He Xue Bao 9: 965-972, 2011.

23. Zhang XJ, Chen YQ, Gui SQ, Qian QH and Guo SX: Clinical observation on Tiangui Capsule for 110 cases of polycystic ovary syndrome. J Trad Chin Med 55: 1835-1840, 2014 (In Chinese).

24. Livak KJ and Schmittgen TD: Analysis of relative gene expression data using real-time quantitative PCR and the 2(-Delta Delta C(T)) method. Methods 25: 402-408, 2001.
25. Fan YS, Sasi R, Lee C, Winter JS, Waterman MR and Lin CC: Localization of the human CYP17 gene (cytochrome P450(17alpha) to $10 \mathrm{q} 24.3$ by fluorescence in situ hybridization and simultaneous chromosome banding. Genomics 14: 1110-1111, 1992.

26. McNatty KP, Makris A, DeGrazia C, Osathanondh R and Ryan KJ: The production of progesterone, androgens, and estrogens by granulosa cells, thecal tissue, and stromal tissue from human ovaries in vitro. J Clin Endocrinol Metab 49: 687-699, 1979.

27. Moon YS, Tsang BK, Simpson C and Armstrong DT: 17beta-Estradiol biosynthesis in cultured granulosa and thecal cells of human ovarian follicles: Stimulation by follicle-stimulating hormone. J Clin Endocrinol Metab 47: 263-267, 1978.

28. Harlow CR, Bradshaw AC, Rae MT, Shearer KD and Hillier SG: Oestrogen formation and connective tissue growth factor expression in rat granulosa cells. J Endocrinol 192: 41-52, 2007.

29. Schulte MM, Tsai JH and Moley KH: Obesity and PCOS: The effect of metabolic derangements on endometrial receptivity at the time of implantation. Reprod Sci 22: 6-14, 2015.

30. Cai D, Yuan M, Frantz DF, Melendez PA, Hansen L, Lee J and Shoelson SE: Local and systemic insulin resistance resulting from hepatic activation of IKK-beta and NF-kappaB. Nat Med 11: 183-190, 2005.

31. Cai D, Frantz JD, Tawa NE Jr, Melendez PA, Oh BC, Lidov HG, Hasselgren PO, Frontera WR, Lee J, Glass DJ and Shoelson SE: IKKbeta/NF-kappaB activation causes severe muscle wasting in mice. Cell 119: 285-298, 2004.

32. Yuan M, Konstantopoulos N, Lee J, Hansen L, Li ZW, Karin M and Shoelson SE: Reversal of obesity- and diet-induced insulin resistance with salicylates or targeted disruption of IKKbeta. Science 293: 1673-1677, 2001.

33. Zhang X, Zhang G, Zhang H, Karin M, Bai H and Cai D: Hypothalamic IKKbeta/NF-kappaB and er stress link overnutrition to energy imbalance and obesity. Cell 135: 61-73, 2008.

34. Benzler J, Ganjam GK, Pretz D, Oelkrug R, Koch CE, Legler K, Stöhr S, Culmsee C, Williams LM and Tups A: Central inhibition of IKK $\beta / \mathrm{NF}-\kappa \mathrm{B}$ signaling attenuates high-fat diet-induced obesity and glucose intolerance. Diabetes 64: 2015-2027, 2015.

35. Mori H, Hanada R, Hanada T, Aki D, Mashima R, Nishinakamura $H$, Torisu T, Chien KR, Yasukawa $\mathrm{H}$ and Yoshimura A: Socs3 deficiency in the brain elevates leptin sensitivity and confers resistance to diet-induced obesity. Nat Med 10: 739-743, 2004.

36. Howard JK, Cave BJ, Oksanen LJ, Tzameli I, Bjorbaek C and Flier JS: Enhanced leptin sensitivity and attenuation of diet-induced obesity in mice with haploinsufficiency of Socs3. Nat Med 10: 734-738, 2004.

This work is licensed under a Creative Commons Attribution-NonCommercial-NoDerivatives 4.0 International (CC BY-NC-ND 4.0) License. 